\title{
WEAK CONVERGENCE OF CONDITIONED BIRTH-DEATH PROCESSES IN DISCRETE TIME
}

\author{
PAULINE SCHRIJNER * AND \\ ERIK A. VAN DOORN ${ }^{\star}$, University of Twente
}

\begin{abstract}
We consider a discrete-time birth-death process on the nonnegative integers with -1 as an absorbing state and study the limiting behaviour as $n \rightarrow \infty$ of the process conditioned on nonabsorption until time $n$. By proving that a condition recently proposed by Martínez and Vares is vacuously true, we establish that the conditioned process is always weakly convergent when all self-transition probabilities are zero. In the aperiodic case we obtain a necessary and sufficient condition for weak convergence.
\end{abstract}

BIRTH-DEATH PROCESS, CONDITIONED PROCESS, RATIO LIMIT, WEAK CONVERGENCE AMS 1991 SUBJECT CLASSIFICATION: PRIMARY 60J80

\section{Introduction}

Roberts and Jacka [3] consider a continuous-time birth-death process on the positive integers with absorption at zero and study the limiting behaviour as $T \rightarrow \infty$ of the process conditioned to remain positive until time $T$. Under the assumptions of nonexplosiveness, irreducibility and certain absorption of the original process, they show that the conditioned process converges weakly to a time-homogeneous birthdeath process. Roberts, Jacka and Pollett [4] have established that the limiting process is nonexplosive.

${ }^{\star}$ Postal address: Faculty of Applied Mathematics, University of Twente, P.O. Box 217, 7500 AE Enschede, The Netherlands 
Recently, Martínez and Vares [2] have addressed similar issues in a discrete-time setting. But their findings are more restrictive than those of Roberts and Jacka [3], their main theorem giving a number of necessary and sufficient conditions for the weak convergence of the conditioned process to a time-homogeneous birth-death process which do not seem easy to verify. However, it will be shown in this note that, solely under the irreducibility condition, weak convergence of the conditioned process to a time-homogeneous birth-death process also occurs in this discrete-time setting, so that each equivalent statement in [2] is actually a theorem.

We note that the discrete-time setting considered by Martínez and Vares [2] involves zero self-transition probabilities. The situation changes drastically when one allows positive self-transition probabilities, for then weak convergence of the conditioned process does not always occur. In fact, we shall derive a necessary and sufficient condition for weak convergence in this case which is closely related to the necessary and sufficient condition for the existence of the limiting conditional distribution of the process given in van Doorn and Schrijner [7].

Crucial in our analysis is a ratio limit theorem for nonabsorption probabilities which complements the ratio limit theorem for transition probabilities in [7].

\section{Preliminaries}

In what follows $X \equiv\{X(n), n=0,1, \ldots\}$ is a homogeneous, discrete-time birthdeath process. It will be convenient to let $\{-1,0,1, \ldots\}$ be the state space of $X$ 
with -1 acting as the absorbing state. We let

$$
p_{i j}(n) \equiv \operatorname{Pr}\{X(m+n)=j \mid X(m)=i\}
$$

and write $p_{i j} \equiv p_{i j}(1)$; in addition we use the shorthand notation $p_{j} \equiv p_{j, j+1}, q_{j+1} \equiv$ $p_{j+1, j}$ and $r_{j} \equiv p_{j j}$. Throughout we assume $p_{j}>0, q_{j}>0$ and $p_{j}+q_{j}+r_{j}=1$ for $j \geq 0$, so that $\{0,1, \ldots\}$ constitutes an open irreducible class. Clearly, this class is periodic with period 2 if $r_{j}=0$ for all $j$, and aperiodic otherwise.

With $X$ we associate the polynomial sequence $\left\{Q_{j}(x)\right\}_{j=0}^{\infty}$ defined by the recurrence relations

$$
\begin{aligned}
& Q_{0}(x)=1, \quad p_{0} Q_{1}(x)=x-r_{0} \\
& x Q_{j}(x)=q_{j} Q_{j-1}(x)+r_{j} Q_{j}(x)+p_{j} Q_{j+1}(x) \quad j \geq 1 .
\end{aligned}
$$

For later use we observe that

$$
Q_{j}(1)=1+q_{0} \sum_{n=0}^{j-1}\left(p_{n} \pi_{n}\right)^{-1} \quad j \geq 1,
$$

where $\pi_{j}$ are constants defined by

$$
\pi_{0} \equiv 1, \quad \pi_{j} \equiv \frac{p_{0} p_{1} \ldots p_{j-1}}{q_{1} q_{2} \ldots q_{j}} \quad j \geq 1
$$

The polynomials $Q_{j}(x), j \geq 0$, are orthogonal with respect to a unique Borel measure $\psi$ on the interval $[-1,1]$, and the transition probabilities $p_{i j}(n)$ can be represented in terms of the polynomials $Q_{j}(x)$ and the measure $\psi$ as

$$
p_{i j}(n)=\pi_{j} \int_{-1}^{1} x^{n} Q_{i}(x) Q_{j}(x) d \psi(x),
$$

see Karlin and McGregor [1] and van Doorn and Schrijner [5, 6]. 


\section{Letting}

$$
\eta \equiv \sup \operatorname{supp}(\psi)
$$

we have $0<\eta \leq 1$ and $\operatorname{supp}(\psi) \subset[-\eta, \eta]$. If the birth-death chain is periodic then the polynomials $Q_{j}(x)$ satisfy

$$
Q_{j}(x)=(-1)^{j} Q_{j}(-x) \quad j \geq 0,
$$

while the associated measure is symmetric about zero. For these results and more information on the polynomials $Q_{j}(x)$ and the associated measure, see $[5,6]$.

Next, we denote by $\tau$ the time to absorption at -1 of $X$ and let

$$
a_{i} \equiv \operatorname{Pr}\{\tau<\infty \mid X(0)=i\}
$$

¿From van Doorn and Schrijner [7] we know

$$
a_{i}=\frac{q_{0} \sum_{k=i}^{\infty}\left(p_{k} \pi_{k}\right)^{-1}}{1+q_{0} \sum_{k=0}^{\infty}\left(p_{k} \pi_{k}\right)^{-1}} \quad i \geq 0
$$

which should be interpreted as 1 if $\sum_{k}\left(p_{k} \pi_{k}\right)^{-1}=\infty$. From (2.2) and (2.5) it follows that

$$
1-a_{i}=\left(1-a_{0}\right) Q_{i}(1) \quad i \geq 0
$$

\section{Ratio limits}

It is shown in [7] that for an aperiodic chain $X$ the limits

$$
\lim _{n \rightarrow \infty} \frac{p_{i k}(n+m)}{p_{j l}(n)} \quad i, j, k, l \geq 0
$$


exist simultaneously if and only if

$$
C_{n}(\psi) \equiv \frac{\int_{-1}^{0}(-x)^{n} d \psi(x)}{\int_{0}^{1} x^{n} d \psi(x)} \rightarrow 0 \text { as } n \rightarrow \infty,
$$

in which case

$$
\lim _{n \rightarrow \infty} \frac{p_{i k}(n+m)}{p_{j l}(n)}=\eta^{m} \frac{Q_{i}(\eta) Q_{k}(\eta)}{Q_{j}(\eta) Q_{l}(\eta)}
$$

In this note our interest focuses on the limits

$$
\lim _{n \rightarrow \infty} \frac{\operatorname{Pr}\{\tau>n+m \mid X(0)=i\}}{\operatorname{Pr}\{\tau>n \mid X(0)=j\}}=\lim _{n \rightarrow \infty} \frac{\sum_{k=0}^{\infty} p_{i k}(n+m)}{\sum_{k=0}^{\infty} p_{j k}(n)}
$$

both in the periodic and the aperiodic case.

\section{Proposition 1}

(i) If absorption is not certain then the limit (3.3) exists for every pair $i, j$ and is given by

$$
\lim _{n \rightarrow \infty} \frac{\operatorname{Pr}\{\tau>n+m \mid X(0)=i\}}{\operatorname{Pr}\{\tau>n \mid X(0)=j\}}=\frac{Q_{i}(1)}{Q_{j}(1)}
$$

(ii) If absorption is certain and $X$ is periodic then the limit (3.3) exists if $\eta=1$, or $\eta<1$ and $m+i+j=$ even, in which case

$$
\lim _{n \rightarrow \infty} \frac{\operatorname{Pr}\{\tau>n+m \mid X(0)=i\}}{\operatorname{Pr}\{\tau>n \mid X(0)=j\}}=\eta^{m} \frac{Q_{i}(\eta)}{Q_{j}(\eta)} .
$$

(iii) If absorption is certain and $X$ is aperiodic then the limit (3.3) exists if $\eta=1$, or $\eta<1$ and $C_{n}(\psi) \rightarrow 0$ as $n \rightarrow \infty$, in which case the limit is given by (3.5); in the opposite case the limit (3.3) does not exist when, e.g., $j=0$ and $i=m=1$. 


\section{Proof.}

(i) If absorption is not certain, then

$$
\lim _{n \rightarrow \infty} \operatorname{Pr}\{\tau>n \mid X(0)=l\}=1-a_{l}>0
$$

so the statement follows with (2.6).

(ii) If absorption is certain, then we know from [7, Eq. (4.6)] that

$$
\sum_{k=0}^{\infty} p_{l k}(n)=1-p_{l,-1}(n)=q_{0} \int_{-1}^{1} x^{n} Q_{l}(x)(1-x)^{-1} d \psi(x) .
$$

In particular, taking $n=l=0$ we see that $q_{0} \int_{-1}^{1}(1-x)^{-1} d \psi(x)=1$, so that $\int_{-1}^{1}\left(1-x^{2}\right)^{-1} d \psi(x)<\infty$. By copying the proof of Lemma 3.1 of [7] it now follows that for any continuous function $f$ on $[0,1]$

$$
\lim _{n \rightarrow \infty} \frac{\int_{0}^{1} x^{n} f(x)\left(1-x^{2}\right)^{-1} d \psi(x)}{\int_{0}^{1} x^{n}\left(1-x^{2}\right)^{-1} d \psi(x)}=f(\eta) .
$$

Since $X$ is periodic we can exploit (2.4) and the symmetry of $\psi$, together with (3.3)

and (3.6), to write

$$
\begin{aligned}
& \frac{\operatorname{Pr}\{\tau>n+m \mid X(0)=i\}}{\operatorname{Pr}\{\tau>n \mid X(0)=j\}} \\
= & \frac{\int_{0}^{1} x^{n+m}\left((-1)^{n+m+i}(1-x)+1+x\right) Q_{i}(x)\left(1-x^{2}\right)^{-1} d \psi(x)}{\int_{0}^{1} x^{n}\left((-1)^{n+j}(1-x)+1+x\right) Q_{j}(x)\left(1-x^{2}\right)^{-1} d \psi(x)} .
\end{aligned}
$$

Finally, dividing numerator and denominator of (3.8) by $\int_{0}^{1} x^{n}\left(1-x^{2}\right)^{-1} d \psi(x)$ and letting $n$ go to infinity through the even integers and through the odd integers, it follows easily from (3.7) that both limits exist, and, moreover, that they are equal 
and given by (3.5) under the stated conditions.

(iii) Since absorption is certain we can apply (3.6) again and write

$$
\frac{\operatorname{Pr}\{\tau>n+m \mid X(0)=i\}}{\operatorname{Pr}\{\tau>n \mid X(0)=j\}}=\frac{\int_{-1}^{1} x^{n+m} Q_{i}(x)(1-x)^{-1} d \psi(x)}{\int_{-1}^{1} x^{n} Q_{j}(x)(1-x)^{-1} d \psi(x)} .
$$

Next dividing both numerator and denominator of (3.9) by $\int_{-1}^{1} x^{n}(1-x)^{-1} d \psi(x)$, we can copy the type of argument leading to Theorem 3.1 in [7], and conclude that (3.5) holds for all $i, j$ and $m$ if and only if

$$
\tilde{C}_{n}(\psi) \equiv \frac{\int_{-1}^{0}(-x)^{n}(1-x)^{-1} d \psi(x)}{\int_{0}^{1} x^{n}(1-x)^{-1} d \psi(x)} \rightarrow 0 \text { as } n \rightarrow \infty,
$$

a counterexample in the opposite case $\left(\tilde{C}_{n}(\psi) \nrightarrow 0\right)$ being provided by $j=0$ and $i=m=1$. We can subsequently write $\tilde{C}_{n}(\psi)$ as the product of three nonnegative factors, viz.

$$
\tilde{C}_{n}(\psi)=C_{n}(\psi) \cdot \frac{\int_{-1}^{0}(-x)^{n}(1-x)^{-1} d \psi(x)}{\int_{-1}^{0}(-x)^{n} d \psi(x)} \cdot \frac{\int_{0}^{1} x^{n} d \psi(x)}{\int_{0}^{1} x^{n}(1-x)^{-1} d \psi(x)},
$$

where the second factor should be interpreted as 1 if $\psi$ has no mass on the negative axis. By the Lemmas 3.3 and 3.4 in [7], $C_{n}(\psi)$ is bounded, while the second factor in (3.11) clearly lies between $1 / 2$ and 1 . Since, by Proposition 3.1 in [7], the third factor tends to $1-\eta$ as $n \rightarrow \infty$, it follows that (3.10) holds if and only if $\eta=1$, or $\eta<1$ and $C_{n}(\psi(x) \rightarrow 0$ as $n \rightarrow \infty$. 


\section{Weak convergence}

Martínez and Vares [2] show that the limits

$$
\lim _{n \rightarrow \infty} \operatorname{Pr}\left\{X(0)=i_{0}, X(1)=i_{1}, \ldots, X(k)=i_{k} \mid \tau>n\right\}
$$

exist for all $k \in N$ and all states $i_{0}, i_{1}, \ldots, i_{k}$ if and only if the limits

$$
\lim _{n \rightarrow \infty} \frac{\operatorname{Pr}\{\tau>n-1 \mid X(0)=j\}}{\operatorname{Pr}\{\tau>n \mid X(0)=i\}}
$$

exist for all pairs $i, j$ such that $p_{i j}>0$, in which case

$$
\begin{array}{r}
\lim _{n \rightarrow \infty} \operatorname{Pr}\left\{X(1)=i_{1}, \ldots, X(k)=i_{k} \mid \tau>n, X(0)=i_{0}\right\} \\
=\operatorname{Pr}\left\{Y(1)=i_{1}, \ldots, Y(k)=i_{k} \mid Y(0)=i_{0}\right\}
\end{array}
$$

where $Y \equiv\{Y(n), n=0,1, \ldots\}$ is the homogeneous birth-death chain on $\{0,1, \ldots\}$

with 1-step transition probabilities $q_{i j} \equiv \operatorname{Pr}\{Y(n+1)=j \mid Y(n)=i\}$ given by

$$
q_{i j}= \begin{cases}p_{i j} \lim _{n \rightarrow \infty} \frac{\operatorname{Pr}\{\tau>n-1 \mid X(0)=j\}}{\operatorname{Pr}\{\tau>n \mid X(0)=i\}} & p_{i j}>0 \\ 0 & p_{i j}=0 .\end{cases}
$$

Relation (4.3) expresses that the process $X$, conditioned on nonabsorption, converges weakly to the process $Y$. With the help of the ratio limit theorem of the previous section we can now establish whether the conditions for weak convergence are satisfied and, if so, what are the 1-step transition probabilities of the limiting process $Y$.

First, if absorption is not certain, Proposition 1 together with (4.4) tell us that the limits (4.2) exist for all $i, j$, and that the 1-step transition probabilities of the limiting process $Y$ satisfy

$$
q_{i j}=\frac{Q_{j}(1)}{Q_{i}(1)} p_{i j} \quad i, j \geq 0 .
$$


In what follows we assume that absorption is certain.

Next looking into the periodic setting considered by Martínez and Vares [2], we have $p_{i i}=r_{i}=0$, so for the conditioned process to converge weakly it is necessary and sufficient that the limits (4.2) exist for all pairs $i, j$ such that $|i-j|=1$. But part (ii) of Proposition 1 together with (4.4) tell us that this is indeed the case, while the 1-step transition probabilities of the limiting process $Y$ are given by

$$
q_{i j}= \begin{cases}\frac{p_{i} Q_{i+1}(\eta)}{\eta Q_{i}(\eta)} & j=i+1, i \geq 0 \\ \frac{q_{i} Q_{i-1}(\eta)}{\eta Q_{i}(\eta)} & j=i-1, i \geq 1 \\ 0 & \text { otherwise. }\end{cases}
$$

(Note that $q_{01}=p_{0} Q_{1}(\eta) / \eta Q_{0}(\eta)=1$ ). We observe that each of the equivalent statements in Theorem 5 of Martínez and Vares [2] is in fact a theorem as a result of this finding.

Finally turning to the aperiodic case we note that for the conditioned process to converge weakly the limits in (4.2) should exist for all pairs $i, j$ such that $|i-j|=1$, or $i=j$ and $r_{i}>0$. Part (iii) of Proposition 1 tells us that this is indeed the case if $\eta=1$; however, if $\eta<1$ the additional condition (3.1) has to be satisfied, in which case the 1-step transition probabilities of the limiting process $Y$ are given by

$$
q_{i j}= \begin{cases}\frac{p_{i} Q_{i+1}(\eta)}{\eta Q_{i}(\eta)} & j=i+1, i \geq 0 \\ \frac{q_{i} Q_{i-1}(\eta)}{\eta Q_{i}(\eta)} & j=i-1, i \geq 1 \\ \frac{r_{i}}{\eta} & j=i, i \geq 0 \\ 0 & \text { otherwise. }\end{cases}
$$


Conversely, if $\eta<1$ and condition (3.1) is not satisfied then Proposition 1 (iii) tells us that for at least one pertinent pair $i, j$ the limit (4.2) does not exist.

We summarize our results in the following theorem.

\section{Theorem 1}

(i) If absorption is not certain then the process $X$, conditioned on nonabsorption, converges weakly to the birth-death process $Y$ with 1-step transition probabilities given by (4.5).

(ii) If absorption is certain and $X$ is periodic then the process $X$, conditioned on nonabsorption, converges weakly to the birth-death process $Y$ with 1-step transition probabilities given by (4.6).

(iii) If absorption is certain and $X$ is aperiodic then the process $X$, conditioned on nonabsorption, converges weakly to the birth-death process $Y$ with 1-step transition probabilities given by (4.7) if and only if either $\eta=1$, or $\eta<1$ and $C_{n}(\psi) \rightarrow 0$ as $n \rightarrow \infty$

\section{Concluding remarks}

If absorption is not certain, it may be more interesting to consider the limits

$$
\lim _{n \rightarrow \infty} \frac{\operatorname{Pr}\{n+m<\tau<\infty \mid X(0)=i\}}{\operatorname{Pr}\{n<\tau<\infty \mid X(0)=j\}}=\lim _{n \rightarrow \infty} \frac{\sum_{k=0}^{\infty} p_{i k}(n+m) a_{k}}{\sum_{k=0}^{\infty} p_{j k}(n) a_{k}}
$$

than the limits (3.3). Doing this we can use [7, Eq. (4.6)] again as a starting point to obtain the following results, in complete analogy with the proofs of Proposition 
1 and Theorem 1.

\section{Proposition 2}

(i) If $X$ is periodic then the limit (5.8) exists if $\eta=1$, or $\eta<1$ and $m+i+j$ is even, in which case

$$
\lim _{n \rightarrow \infty} \frac{\operatorname{Pr}\{n+m<\tau<\infty \mid X(0)=i\}}{\operatorname{Pr}\{n<\tau<\infty \mid X(0)=j\}}=\eta^{m} \frac{Q_{i}(\eta)}{Q_{j}(\eta)}
$$

(ii) If $X$ is aperiodic then the limit (5.8) exists if $\eta=1$, or $\eta<1$ and $C_{n}(\psi) \rightarrow 0$ as $n \rightarrow \infty$, is which case the limit is given by (5.9); in the opposite case the limit (5.8) does not exist when, e.g., $j=0$ and $i=m=1$.

\section{Theorem 2}

(i) If $X$ is periodic then the process $X$, conditioned on the event that absorption has not yet occurred but will occur eventually, converges weakly to the birthdeath process $Y$ with 1-step transition probabilities given by (4.6).

(ii) If $X$ is aperiodic then the process $X$, conditioned on the event that absorption has not yet occurred but will occur eventually, converges weakly to the birthdeath process $Y$ with 1-step transition probabilities given by (4.7) if and only if either $\eta=1$, or $\eta<1$ and $C_{n}(\psi) \rightarrow 0$ as $n \rightarrow \infty$.

We conclude with two observations. First the transition rates (4.7) (which reduce to (4.6) in the periodic case) of the limiting process $Y$, define a birth-death process which is precisely the Derman - Vere-Jones transformation of the original process $X$, see [6] for a definition and further properties. Secondly, when $X$ is aperiodic 
and $\eta<1$, then the necessary and sufficient condition for weak convergence of the conditioned process is identical to the necessary and sufficient condition for the convergence, as $n \rightarrow \infty$, of the conditional probabilities

$$
\operatorname{Pr}\{X(n)=j \mid X(0)=i, n<\tau<\infty\} \quad j \geq 0,
$$

to an honest probability distribution, see [7].

Acknowledgement. We thank Phil Pollett for sending us reference [4] prior to publication and Masaaki Kijima for his comments on the first draft of this paper.

\section{References}

[1] Karlin, S. and McGregor, J.L. (1959). Random walks. Illinois J. Math. 3, 66-81.

[2] Martínez, S. and Vares, M.E. (1995). A Markov chain associated with the minimal quasi-stationary distribution of birth-death chains. J. Appl. Probab. 32, 25-38.

[3] Roberts, G.O. and Jacka, S.D. (1994). Weak convergence of conditioned birth and death processes. J. Appl. Probab. 31, 90-100.

[4] Roberts, G.O., Jacka, S.D. and Pollett, P.K. (1995). Non-explosivity of limits of conditioned birth and death processes. J. Appl. Probab., to appear.

[5] van Doorn, E.A. and Schrijner, P. (1992). Random walk polynomials and random walk measures. J. Comput. Appl. Probab. 49, 289-296.

[6] van Doorn, E.A. and Schrijner, P. (1995). Geometric ergodicity and quasistationarity in discrete-time birth-death processes. J. Austral. Math. Soc. Ser. B 37, 1-24.

[7] van Doorn, E.A. and Schrijner, P. (1995). Ratio limits and limiting conditional distributions for discrete-time birth-death processes. J. Math. Anal. Appl. 190, 263-284. 